\title{
On the Divisibility of Character Values of the Symmetric Group
}

\author{
Jyotirmoy Ganguly Amritanshu Prasad* \\ The Institute of Mathematical Sciences (HBNI) \\ CIT Campus, Taramani, Chennai 600113, India \\ \{jyotirmoy, amri\}@imsc.res.in \\ Steven Spallone \\ Department of Mathematics \\ Indian Institute of Science Education and Research \\ Homi Bhabha Road, Pashan, Pune 411008, India \\ sspallone@iiserpune.ac.in
}

Submitted: Apr 26, 2019; Accepted: Mar 13, 2020; Published: Apr 3, 2020

(C) The authors. Released under the CC BY-ND license (International 4.0).

\begin{abstract}
Fix a partition $\mu=\left(\mu_{1}, \ldots, \mu_{m}\right)$ of an integer $k$ and positive integer $d$. For each $n \geqslant k$, let $\chi_{\mu}^{\lambda}$ denote the value of the irreducible character $\chi^{\lambda}$ of $S_{n}$, corresponding to a partition $\lambda$ of $n$, at a permutation with cycle type $\left(\mu_{1}, \ldots, \mu_{m}, 1^{n-k}\right)$. We show that the proportion of partitions $\lambda$ of $n$ such that $\chi_{\mu}^{\lambda}$ is divisible by $d$ approaches 1 as $n$ approaches infinity.
\end{abstract}

Mathematics Subject Classifications: 20C30, 05A16, 05A17

\section{Introduction}

Let $k$ be a positive integer, and $\mu=\left(\mu_{1}, \ldots, \mu_{m}\right)$ a partition of $k$. For a partition $\lambda$ of an integer $n \geqslant k$, let $\chi_{\mu}^{\lambda}$ denote the value of the irreducible character of $S_{n}$ corresponding to $\lambda$ at an element with cycle type $\left(\mu_{1}, \ldots, \mu_{m}, 1^{n-k}\right)$. The purpose of this article is to prove: Main Theorem. For any positive integers $k$ and $d$, and any partition $\mu$ of $k$,

$$
\lim _{n \rightarrow \infty} \frac{\#\left\{\lambda \vdash n \mid \chi_{\mu}^{\lambda} \text { is divisible by } d\right\}}{p(n)}=1 .
$$

Here $p(n)$ denotes the number of partitions of $n$.

\footnotetext{
*Supported by a Swarnajayanti Fellowship of the Department of Science \& Technology, Govt. of India.
} 
In particular, for any integer $d$, the probability that an irreducible character of $S_{n}$ has degree divisible by $d$ converges to 1 as $n \rightarrow \infty$.

Recall the theorem of Lassalle $\left[4\right.$, Theorem 6], which defines a rational number $A_{\mu}^{\lambda}$ such that

$$
\chi_{\mu}^{\lambda}=\frac{f_{\lambda}}{(n)_{k}} A_{\mu}^{\lambda}
$$

Here $(n)_{k}=n(n-1) \cdots(n-k+1)$, and $f_{\lambda}$ is the degree of the irreducible character of $S_{n}$ corresponding to $\lambda$.

In fact, $A_{\mu}^{\lambda}$ is an integer for all $\lambda, \mu$. This is likely well known to experts, but for the convenience of the reader we sketch a proof in Section 4.

From here, in order to prove the main theorem, we focus on the divisibility properties of $f_{\lambda}$. For each prime number $q$, let $v_{q}(m)$ denote the $q$-adic valuation of an integer $m$, in other words, $q^{v_{q}(m)}$ is the largest power of $q$ that divides $m$. The main theorem will follow from the following result:

Theorem A. For every prime number $q$ and non-negative integer $m$,

$$
\lim _{n \rightarrow \infty} \frac{\#\left\{\lambda \vdash n \mid v_{q}\left(f_{\lambda}\right) \leqslant m+(q-1) \log _{q} n\right\}}{p(n)}=0 .
$$

In the rest of this article, we first prove Theorem A, next show that it implies the main theorem, and then explain the integrality of $A_{\mu}^{\lambda}$.

\section{Proof of Theorem A}

The proof of Theorem A is based on the theory of $q$-core towers. This construction originated in the seminal paper [5] of Macdonald, and was developed further by Olsson in [6]. We now recall the relevant aspects.

Let $[q]$ denote the set $\{0, \ldots, q-1\}$. Consider the disjoint union

$$
T_{q}=\coprod_{i=0}^{\infty}[q]^{i}=\left\{\left(a_{1}, \ldots, a_{i}\right) \mid i \in \mathbf{N}, a_{i} \in[q]\right\} \cup\{\emptyset\},
$$

which can be regarded as a rooted $q$-ary tree with root $\emptyset$ as follows. The children of a vertex $\left(a_{1}, \ldots, a_{i}\right) \in[q]^{i}$ are the vertices $\left(a_{1}, \ldots, a_{i}, a_{i+1}\right)$, where $a_{i+1} \in[q]$. For $q=2$, rows 0 to 3 of this tree are as below. For compactness, commas and parentheses have been omitted.

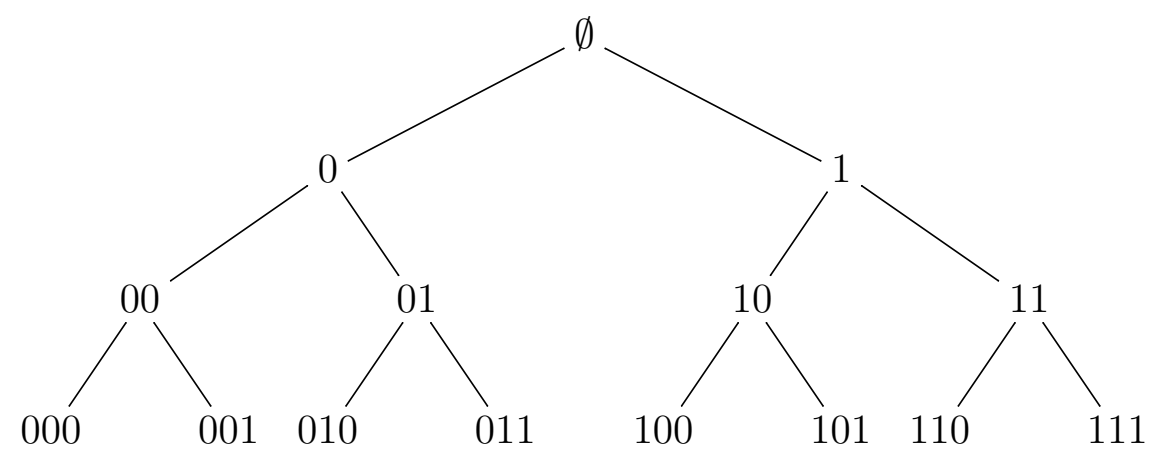


A partition $\lambda$ is said to be a $q$-core if no cell in its Young diagram has hook length divisible by $q$. Denote the set of all $q$-core partitions by $C_{q}$. The $q$-core tower construction associates to each partition $\lambda$ of $n$ a function $\mathcal{T}_{q}^{\lambda}: T_{q} \rightarrow C_{q}$ known as the $q$-core tower of $\lambda$ (see [6, pages 29-30]). In particular, $\mathcal{T}_{q}^{\lambda}(\emptyset)$ is the $q$-core of $\lambda$ (the partition obtained by removing all the $q$-hooks from the Young diagram of $\lambda$ ). This function is visualized as the rooted $q$-ary tree $T_{q}$ with each vertex $x$ of $T_{q}$ replaced by the partition $\mathcal{T}_{q}^{\lambda}(x) \in C_{q}$. For example, the 2-core tower of $\lambda=(4,3,2,2,2,2)$ is:
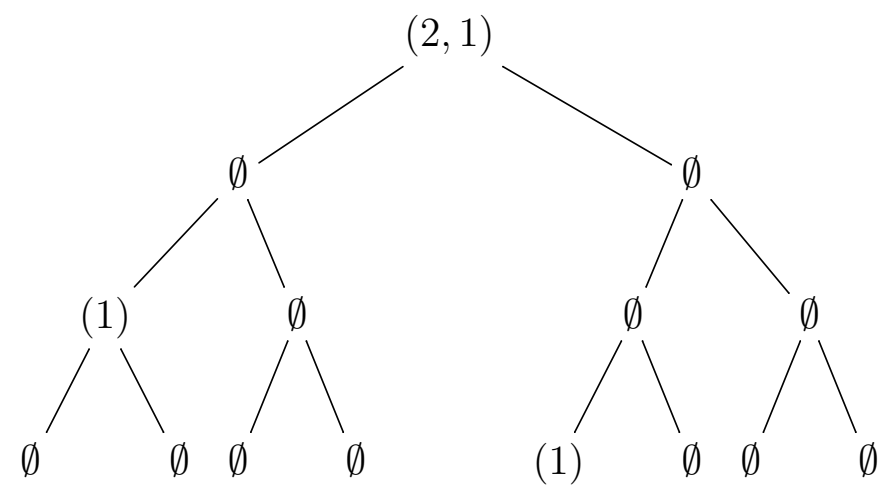

For a function $\mathcal{T}_{q}: T_{q} \rightarrow C_{q}$, define:

$$
w_{i}\left(\mathcal{T}_{q}\right)=\sum_{x \in[q]^{i}}\left|\mathcal{T}_{q}(x)\right|
$$

Then the $q$-core tower satisfies the following constraint:

$$
\sum_{i=0}^{\infty} w_{i}\left(\mathcal{T}_{q}^{\lambda}\right) q^{i}=n
$$

In particular, $\mathcal{T}_{q}^{\lambda}(x)=\emptyset$ for all $i>\log _{q} n$. This function $\lambda \mapsto \mathcal{T}_{q}^{\lambda}$ is a bijection from the set of partitions of $n$ onto the set of all functions $\mathcal{T}: T_{q} \rightarrow C_{q}$ satisfying the condition

$$
\sum_{i=0}^{\infty} w_{i}(\mathcal{T}) q^{i}=n
$$

Define the weight of a $q$-core tower $\mathcal{T}_{q}$ as:

$$
w\left(\mathcal{T}_{q}\right)=\sum_{i=0}^{\infty} w_{i}\left(\mathcal{T}_{q}\right) .
$$

For a partition $\lambda$, define $w(\lambda)=w\left(\mathcal{T}_{q}^{\lambda}\right)$.

Let $n$ be a positive integer with $q$-ary expansion:

$$
n=a_{0}+a_{1} q+\cdots+a_{r} q^{r}, \text { with } a_{i} \in[q] \text { for } i=1, \ldots, r \text {, and } a_{r}>0 .
$$

Define $a(n)=\sum_{i=0}^{r} a_{i}$.

Recall the following Theorem: 
Theorem 1 ([5, Equation (3.3)]). For any partition $\lambda$ of $n$ and any prime $q$,

$$
v_{q}\left(f_{\lambda}\right)=\frac{w(\lambda)-a(n)}{q-1} .
$$

For example when $\lambda=(4,3,2,2,2,2)$, a partition of 15 , and $q=2$, from the 2-core tower of $\lambda$ computed above, $w_{1}(\lambda)=3, w_{2}(\lambda)=0, w_{3}(\lambda)=w_{4}(\lambda)=1$ so that $w(\lambda)=5$. On the other hand $a(15)=4$. Therefore $v\left(f_{\lambda}\right)=w(\lambda)-a(n)=1$.

Theorem 1 can be used to find constraints on partitions with small values of $v_{q}\left(f_{\lambda}\right)$. Suppose that $v_{q}\left(f_{\lambda}\right) \leqslant b$. By Theorem 1 , this is equivalent to

$$
w(\lambda) \leqslant a(n)+b(q-1) .
$$

The expansion $(*)$ implies that $r \leqslant \log _{q} n<r+1$, so that $a(n) \leqslant(q-1)(r+1) \leqslant$ $(q-1)\left(\log _{q} n+1\right)$. So if $v_{q}\left(f_{\lambda}\right) \leqslant b$, then

$$
w(\lambda) \leqslant(q-1)\left(\log _{q} n+1+b\right) .
$$

Thus an upper bound for the number $p_{b}(n)$ of partitions $\lambda$ of $n$ such that $v_{q}\left(f_{\lambda}\right) \leqslant b$ can be obtained by counting the number of $q$-core towers with weight $(q-1)\left(\log _{q} n+1+b\right)$ or less. The total number of vertices in the first $r+1$ rows of $T_{q}$, i.e., in $\coprod_{i=0}^{r}[q]^{i}$, is:

$$
1+q+\cdots+q^{r}=\frac{q^{r+1}-1}{q-1}<q n,
$$

since $q^{r} \leqslant n$. Let $c_{q}(n)$ denote the number of $q$-core partitions of $n$. Set $N_{b}=(q-$ 1) $\left(\log _{q} n+b+1\right)$. Let $\tilde{c}_{q}(n)$ denote $\max \left\{c_{q}(i) \mid 1 \leqslant i \leqslant n\right\}$. There are $\left(\begin{array}{c}w+N-1 \\ w\end{array}\right)$ ways to distribute the weight $w$ into $N$ vertices of $T_{q}$. Thus

$$
\begin{aligned}
p_{b}(n) & \leqslant \tilde{c}_{q}\left(N_{b}\right)^{N_{b}}\left(\begin{array}{c}
q n+N_{b} \\
N_{b}
\end{array}\right) \\
& \leqslant \tilde{c}_{q}\left(N_{b}\right)^{N_{b}}\left(q n+N_{b}\right)^{N_{b}}
\end{aligned}
$$

It is known that, for every integer $q$, there exists a polynomial $f_{q}(n)$ such that $\tilde{c}_{q}(n) \leqslant$ $f_{q}(n)$ for all $n \geqslant 0$. Indeed, for $q=2$, it is well-known that $c_{2}(n) \leqslant 1$, and for $q=3$, using a formula of Granville and Ono [2, Section 3, p. 340], $c_{3}(n) \leqslant 3 n+1$. For $q \geqslant 4$, the existence of $f_{q}(n)$ follows from Anderson [1, Corollary 7].

We get:

$$
p_{b}(n) \leqslant f_{q}\left(N_{b}\right)^{N_{b}}\left(q n+N_{b}\right)^{N_{b}},
$$

whence

$$
\log _{q} p_{b}(n) \leqslant N_{b}\left[\log _{q} f_{q}\left(N_{b}\right)+\log _{q}\left(q n+N_{b}\right)\right] .
$$

Taking $b=m+(q-1) \log _{q} n$ gives $N_{b}=(q-1)\left(q \log _{q} n+m+1\right)$. Thus $\log _{q} p_{b}(n)=o\left(n^{\epsilon}\right)$ for every $\epsilon>0$. On the other hand, the Hardy-Ramanujan asymptotic [3] for $p(n)$ implies that $\log _{q} p(n)$ grows faster than $n^{\frac{1}{2}-\epsilon}$ for any $\epsilon>0$. Thus Theorem A follows. 


\section{Proof of the Main Theorem}

The identity (1) implies that

$$
v_{q}\left(\chi_{\mu}^{\lambda}\right) \geqslant v_{q}\left(f_{\lambda}\right)-v_{q}\left((n)_{k}\right)
$$

Using Legendre's formula on the valuation of a factorial, that $v_{q}(n !)=\frac{n-a(n)}{q-1}$, we have:

$$
v_{q}\left((n)_{k}\right)=v_{q}\left(\frac{n !}{(n-k) !}\right)=\frac{k+a(n-k)-a(n)}{q-1} \leqslant k+(q-1) \log _{q} n .
$$

Hence if $v_{q}\left(f_{\lambda}\right) \geqslant m+(q-1) \log _{q} n$, then $v_{q}\left(\chi_{\mu}^{\lambda}\right) \geqslant(m-k)$. Thus taking $m=k+b$ in Theorem A tells us that

$$
\lim _{n \rightarrow \infty} \frac{\#\left\{\lambda \vdash n \mid v_{p}\left(\chi_{\mu}^{\lambda}\right) \leqslant b\right\}}{p(n)}=0 .
$$

From this the main theorem follows.

\section{Integrality of $A_{\mu}^{\lambda}$}

For each partition $\mu=\left(\mu_{1}, \ldots, \mu_{m}\right)$, with $m$ positive parts, the constant $A_{\mu}^{\lambda}$ from (1) is given in $[4$, Theorem 6$]$ as

$$
A_{\mu}^{\lambda}=\sum_{\varepsilon} \sum_{\left(i_{1}, \ldots, i_{m}\right)} A_{i_{1}, \ldots, i_{m}}^{(\varepsilon)}(\mu) \prod_{k} c_{i_{k}}^{\lambda}\left(\mu_{k}\right) .
$$

The $\varepsilon$ in the first sum runs over "upper triangular matrices" $\varepsilon=\left(\varepsilon_{i j}\right)$ for $1 \leqslant i<j \leqslant n$ with $\varepsilon_{i j} \in\{0,2\}$. The second sum runs over $r$-tuples of nonnegative integers. The quantities $c_{i}^{\lambda}(q)$, defined for nonnegative integers $i, q$ and partitions $\lambda$, are certain rational numbers. Their "boundary values" are $c_{0}^{\lambda}(q)=-1 / q$, and $c_{i}^{\emptyset}(q)=0$ for $i>0$. However, $c_{i}^{\lambda}(q)$ are integers for $i>0$, which may be seen recursively by Lemma 1 on page 396 of [4]. The quantities $A_{i_{1}, \ldots, i_{m}}^{(\varepsilon)}(\mu)$ are defined in Theorem 6 on page 399 by an intricate formula.

For a given $\varepsilon$ and $i_{1}, \ldots, i_{m}$, we argue below that each of the terms

$$
A_{i_{1}, \ldots, i_{m}}^{(\varepsilon)}(\mu) \cdot \prod_{k} c_{i_{k}}^{\lambda}\left(\mu_{k}\right)
$$

from (3) are integers. From this it follows that $A_{\mu}^{\lambda}$ is an integer.

Lemma 2. If there exists a $k$ with

1. $\varepsilon_{\ell k}=0$ for all $\ell<k$,

2. $\varepsilon_{k \ell}=0$ for all $k<\ell$, and 
3. $i_{k}=0$,

then

$$
A_{i_{1}, \ldots, i_{m}}^{(\varepsilon)}\left(\mu_{1}, \ldots, \mu_{m}\right)=0 .
$$

Proof. In the definition of $A_{i_{1}, \ldots, i_{m}}^{(\varepsilon)}\left(\mu_{1}, \ldots, \mu_{m}\right)$ in Theorem 6 of Lassalle, it is expressed as a sum over certain $a, b$ of products, including a product over certain Stirling numbers. The Stirling number corresponding to $k$ as above is

$$
s\left(\mu_{k}+1,0\right)=0,
$$

since by the given convention the $a$ 's and $b$ 's are 0 .

Let $Z=\left\{k: i_{k}=0\right\}$. The product $\prod_{k} c_{i_{k}}^{\lambda}\left(\mu_{k}\right)$ is an integer multiple of $\prod_{k \in Z} \mu_{k}^{-1}$, and $A_{i_{1}, \ldots, i_{m}}^{(\varepsilon)}(\mu)$ is an integer multiple of

$$
\prod_{i<j} \theta_{i j}=\prod_{i<j \text { and } \varepsilon_{i j} \neq 0} \mu_{i} \mu_{j}
$$

Therefore $A_{i_{1}, \ldots, i_{m}}^{(\varepsilon)}(\mu) \cdot \prod_{k} c_{i_{k}}^{\lambda}\left(\mu_{k}\right)$ is an integer, unless for some $k \in Z$ we have $\varepsilon_{i k}=0$ for all $i<k$ and $\varepsilon_{k j}=0$ for all $k<j$. But then the product anyway vanishes by Lemma 2 .

Since $A_{\mu}^{\lambda}$ is the sum over the tuples $\left(i_{1}, \ldots, i_{m}\right)$ and the $(\varepsilon)$ of the $A_{i_{1}, \ldots, i_{m}}^{(\varepsilon)}(\mu) \cdot \prod_{k} c_{i_{k}}^{\lambda}\left(\mu_{k}\right)$, it too must be an integer.

\section{Acknowledgements}

The authors thank the referee for several helpful remarks, which helped improve the readability of this article.

\section{References}

[1] J. Anderson. An asymptotic formula for the $t$-core partition function and a conjecture of Stanton. J. Number Theory, 128(9):2591-2615, 2008.

[2] A. Granville and K. Ono. Defect zero $p$-blocks for finite simple groups. Trans. Amer. Math. Soc., 348(1):331-347, 1996.

[3] G. H. Hardy and S. Ramanujan. Asymptotic formulae in combinatory analysis. Proc. London Math. Soc. (2), 17(1):75-115, 1918.

[4] M. Lassalle. An explicit formula for the characters of the symmetric group. Math. Ann., 340(2):383-405, 2008.

[5] I. G. Macdonald. On the degrees of the irreducible representations of symmetric groups. Bull. London Math. Soc., 3:189-192, 1971.

[6] J. B. Olsson. McKay numbers and heights of characters. Math. Scand., 38(1):25-42, 1976. 